\title{
Digital Heart Manual: making technology accessible with a user-friendly resource
}

\author{
Dr Carolyn Deighan \\ The Heart Manual Department \\ Astley Ainslie Hospital, Edinburgh \\ Carolyn.Deighan@nhslothian.scot.nhs.uk
}

Jenny Elliott

The Heart Manual Department Astley Ainslie Hospital, Edinburgh

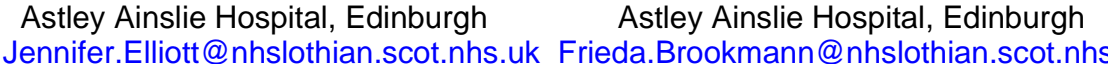

\author{
Dr Claudia Pagliari \\ Centre for Population Health Sciences \\ University of Edinburgh \\ claudia.pagliari@ed.ac.uk \\ es
}

Frieda Brookmann

The Heart Manual Department
Lucie Michalova

The Heart Manual Department

Astley Ainslie Hospital, Edinburgh
lucie.michalova@nhslothian.scot.nhs.uk

Louise Taylor

The Heart Manual Department

Astley Ainslie Hospital, Edinburgh

Louise.Taylor@nhslothian.scot.nhs.uk

\begin{abstract}
The Heart Manual (HM) NHS Lothian, UK's leading home-based cardiac rehabilitation programme. In collaboration with e-health experts, patients and health professionals an innovative digital version of the original programme was developed and further refined using input and expertise of cardiac rehabilitation patient graduates and health professionals. Usage was monitored, and usability and accessibility assessed. Participants fed back over a number of iterations of the resource. The evaluation has highlighted the perceived user-friendliness of the HM digital format and its effectiveness in delivering the key messages of the programme. It also flagged several areas for further modification of the resource. Our pilot project has emphasised the value of employing a user-centred approach when developing and improving web based interventions. Such approach should result in better matched interventions which will maximise patients' engagement and received benefits.
\end{abstract}

Heart Manual, digital intervention, cardiac rehabilitation, usability.

\section{INTRODUCTION}

Health service users seek greater choice and flexibility in how they engage with self-management programmes. Although innovative digital resources may promote engagement in cardiac rehabilitation, it is often unclear how usable and acceptable these interventions are for their prospective users. Usability and accessibility problems often mean that evidenced based web intervention are not being implemented, underutilising its potential to positively affect patients' health outcomes.

Heart Manual (HM) NHS Lothian is the UK's leading home-based cardiac rehabilitation programme, evidenced by three RCTs over 20 years [1-3]. The $\mathrm{HM}$ is an excellent example of a facilitated, sustainable and comprehensive resource used by multi-disciplinary teams throughout the UK and internationally. In collaboration with e-health experts, patients and health professionals we have developed a digital version of the original programme.

The aim of the current project was to evaluate the acceptability, accessibility and usability of the new digital format for cardiac patients, their families and health professionals.

\section{DIGITAL HEART MANUAL}

The innovative digital version of the $\mathrm{HM}$ programme has been developed with support from the University of Edinburgh, Edinburgh and Lothians Health Foundation, the Scottish Government, Edinburgh College, Scottish Health on the Web (SHOW) and Edinburgh Chamber of Commerce to meet the Scottish Government's innovation strategy. It has been constructed with input from a multi-disciplinary team made up of nurses, psychologists, web developers/designers and eHealth experts. It was crucial to retain the effectiveness of the original $\mathrm{HM}$ in an easy to use, engaging digital format.

The digital HM was developed with the aim to:

- Improve access of cardiac rehabilitation services to younger people diagnosed with myocardial infarction or undergoing revascularisation;

- Provide greater support for people returning to work; providing opportunities to integrate the HM into working life earlier after a heart attack or revascularisation;

- Engage with greater number of people using digital technology in day-to-day living, including mobile platforms for online health;

- Improve adaptability of manual to other languages and conditions and

- Provide health professionals with more flexibility in intervention delivery. 


\section{METHODOLOGY}

The study adopted an "agile development approach," which guided the entire process of developing and improving the innovative digital version of the HM Programme. A range of prospective users (health professionals and patients familiar/unfamiliar with the original HM or other hospital based programmes) were invited to access the digital prototype and test its usability. Confidence with technology, usability and acceptability was assessed with a semi-structured Usability and Accessibility questionnaire derived from the standardised Web Content Accessibility Guidelines (WCAG) 2.0. [4-5]. A tracking system remotely collected data on participants' usage. Thematic analysis of qualitative data was undertaken, leading to suggestions for improvements to the resource. Once these improvements were made, follow-up interviews helped add to and further develop themes.

\section{FINDINGS}

In total 28 people participated in the evaluation, including 17 patient representatives aged 42 - 77 and 11 health professionals. Usage data highlighted how the resource fitted in with participants' daily lives. Four key themes emerged from the analysis of the qualitative data, namely concept of the digital manual, barriers to use, perceived usability and content. These were further developed in follow-up interviews.

The analysis revealed the digital HM was viewed as well structured, clearly presented and easy to use. It was apparent that the digital format was acceptable for a range of ages, challenging our assumptions about who might benefit and engage with this digital intervention. Patient users also highlighted the depth and quality of the content and the value of the extensive psychological component which those new to the HM would have welcomed in the aftermath of their previous cardiac event. The interactive physical activity component was also seen to be a useful aid to self management post heart attack.

Moreover, the user-centred feedback identified a number of potential improvements, such as the benefits of incorporating links and features to maximise the accessibility of content and the need to provide more flexible and intuitive navigation pathways. These suggestions informed the final version of the resource.

\section{CONCLUSION}

The current project has highlighted the value of engaging patients as well as health professionals in the process of developing and refining web-based health interventions. Such an approach has proved to be a mutually beneficial experience and has resulted in a digital intervention which is usable and acceptable for users, despite their differences in age and confidence with technology. Better matched digital cardiac rehabilitation programmes are more likely to encourage patient engagement and maximise received benefits.

\section{REFERENCES}

[1] Clark, M., Kelly, T., Deighan, C. (2011) A systematic review of the Heart Manual literature. Eur J Cardiovasc Nurs, 10, 3-13.

[2] Dalal, H. M., Zawada, A., Jolly, K., Moxham, T., Taylor, R. S. (2010) Home based vs. centre based cadiac rehabilitation: Cochrane systematic review and meta-analysis. BMJ, 340, b5631.

[3] McClure, T., Haykowsky, M. J., Schopfolcher, D., Hsu, Zy., Clark, A. M. (2013) Home-based secondary prevention programs for patients with coronary artery disease: a meta-analysis of effects on anxiety.

[4] World Wide Web Consortium. (2008) Web content accessibility guidelines (WCAG) 2.0. W3C. http://www.w3.org/TR/WCAG20/.

[5] World Wide Web Consortium. (2010) Web accessibility for older users: a literature review. http://www.w3.org/TR/wai-age-literature/. 